\title{
Modified generalized beam lattice model associated with fracture of reinforced fiber/particle composites
}

\author{
J.X. Liu ${ }^{\text {a,* }}$, Z.Y. Zhao ${ }^{\text {a }}$, S.C. Deng ${ }^{b}$, N.G. Liang ${ }^{c}$ \\ a PTRC, School of Civil and Environmental Engineering, Nanyang Technological University, Nanyang Avenue 50, Singapore 639798, Singapore \\ ${ }^{\mathrm{b}}$ Laboratory of High Temperature Gas Dynamics (LHD), Institute of Mechanics, Chinese Academy of Sciences, 100080 Beijing, China \\ ' State Key Laboratory of Nonlinear Mechanics (LNM), Institute of Mechanics, Chinese Academy of Sciences, 100080 Beijing, China
}

\section{A R T I C L E I N F O}

\section{Article history:}

Available online 24 July 2008

\section{Keywords:}

Generalized beam lattice model

Fracture analyses

Reinforced fiber/particle composite

Heterogeneity

Numerical simulation

\begin{abstract}
A B S T R A C T
Lattice-type model can simulate in a straightforward manner heterogeneous brittle media. Mohr-Coulomb failure criterion has recently been involved into the generalized beam (GB) lattice model, and as a result, numerical experiments on concrete under various loading conditions can be conducted. The GB lattice model is further used to investigate the reinforced fiber/particle composites instead of only particle composites as the model did before. Numerical examples are given to show the effectiveness of the modeling procedure, and influences of inclusions (particle, fiber and rebar) on the fracture processes are also discussed.
\end{abstract}

(c) 2008 Elsevier Ltd. All rights reserved.

\section{Introduction}

When investigating fracture behaviors in heterogeneous media such as concrete in the macro continuum level, there are mainly two kinds of numerical modeling: discrete crack and smeared crack models [1-3]. The discrete crack model is aimed at simulating the initiation and propagation of dominant cracks. When the nodal stress at the node ahead of the crack tip introduced as a geometric entity exceed the tensile strength, the node is split into two nodes and the tip of the crack is assumed to propagate to the next node. The successive adjustment in mesh topology is a prominent difficulty in discrete crack model. In contrast, the smeared crack model is based on the idea that in concrete, due to its heterogeneity and the presence of reinforcement, many small (micro-) cracks nucleate and grow part of which link up to form dominant crack(s) only in a later stage of the loading process. Since each individual crack is not numerically resolved, the smeared crack model captures the degrading process through a constitutive relation, thus smearing out the cracks over the continuum. The smearing operation is actually based on the basic assumption of micro-mechanics, i.e., "the doctrine that all observable events must be explained as macro events; that is to say, as averages or summations of certain micro events" [4]. However, the validity of this doctrine may become debatable if the disorder on a finer scale affects the response on the coarse scale in a manner that is not related to "averages or

\footnotetext{
* Corresponding author.

E-mail address: liux0014@ntu.edu.sg (J.X. Liu).
}

summations". In principle, this questionable issue also puzzles the extended finite element method (X-FEM) proposed in [5,6].

In comparison to the above macro level models, the meso-level lattice-type model can simulate the complete failure process including nucleation and growth of micro cracks, formation, coalition and propagation of macro cracks until the final breakdown of the whole specimen [7-9]. Furthermore, this kind of models does not need any remeshing procedure or any smearing operation. Most important of all, lattice models describe the failure process in a very realistic and physical-based manner: successive cracks of matrix, aggregate and interface units, showing clearly the relation between microstructure and failure behavior. Of course, the computational command will be higher. Fortunately, the limit from the computational conditions has been and is being substantially overcome by quick development of computer hardware.

In fact, lattice-type models have been successfully used in fracture analyses for several decades. The GB lattice model proposed recently was employed to simulate tensile failures by adopting maximum tensile stress failure criterion at first [9]. Then it has been improved to investigate compressive failures by using the Mohr-Coulomb criterion [10]. The Mohr-Coulomb criterion is widely accepted to describe elemental failing behavior under various loading conditions. Therefore, the GB lattice model improved in [10] can simulate a wider range of fracture experiments in principle.

Until now, the media investigated using lattice-type models are mainly particle composites [7-10]. Nevertheless, fibers and rebar are another two common kinds of inclusions in many engineering composites such as concrete. Therefore, it is also an interesting 
topic to investigate fracture behaviors of composites containing fibers and rebar, which is the aim in the present paper.

\section{Modeling and algorithm}

\subsection{Generalized beam lattice model and its extension into reinforced fiber/particle composites}

To take into account the heterogeneity nature of concrete, the GB lattice model has been developed [9]. Until now, the concerns in the literature have been mainly focused on the inclusion of aggregates, i.e., particles (Fig. 1a). Every GB element is composed of three beams, which can be aggregate-phase, matrix-phase or interface-phase independently (Fig. 1b). In the three-fragment GB element, every fragment is regarded as a beam. These beams can be taken as Euler-Bernoulli beams, Timoshenko beams or beams proposed by [11]. No matter which beam theory is adopted, the beam stiffness matrices can be expressed in the following common form (Fig. 2):

$$
\left\{\begin{array}{l}
Q_{1} \\
N_{1} \\
M_{1} \\
Q_{2} \\
N_{2} \\
M_{2}
\end{array}\right\}=\underbrace{\left[\begin{array}{cccccc}
M_{11} & 0 & -M_{34} & -M_{11} & 0 & -M_{34} \\
& M_{22} & 0 & 0 & -M_{22} & 0 \\
& & M_{33} & M_{34} & 0 & M_{36} \\
& & & M_{11} & 0 & M_{34} \\
& & S Y M & & M_{22} & 0 \\
& & & & & M_{33}
\end{array}\right]}_{M}\left\{\begin{array}{l}
u_{1} \\
v_{1} \\
\varphi_{1} \\
u_{2} \\
v_{2} \\
\varphi_{2}
\end{array}\right\}
$$

where, $\mathbf{F}_{12}=\left\{Q_{1} N_{1} M_{1} Q_{2} N_{2} M_{2}\right\}^{\mathrm{T}}$ and $\mathbf{u}_{12}=\left\{\begin{array}{llllll}u_{1} & v_{1} & \varphi_{1} & u_{2} & v_{2} & \varphi_{2}\end{array}\right\}^{\mathrm{T}}$ are the generalized force vector and the generalized displacement vector, respectively.

For the Euler-Bernoulli beam:

$$
\begin{array}{ll}
M_{11}=\frac{12 E^{(b)} I}{h^{3}}, & M_{34}=\frac{6 E^{(b)} I}{h^{2}}, \quad M_{22}=\frac{E^{(b)} A}{h}, \\
M_{33}=\frac{4 E^{(b)} I}{h}, & M_{36}=\frac{2 E^{(b)} I}{h}
\end{array}
$$

a

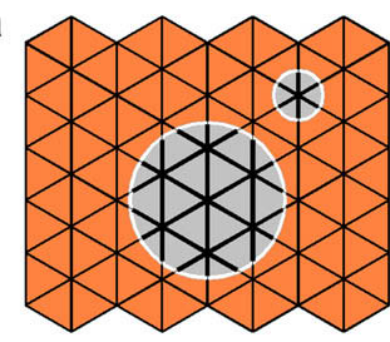

b

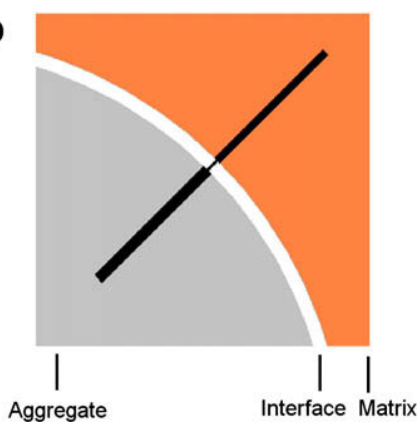

Fig. 1. A GB lattice for particle composites: (a) projection of particle structure onto the lattice and (b) sketch-map of composition of an aggregate-interface-matrix element.

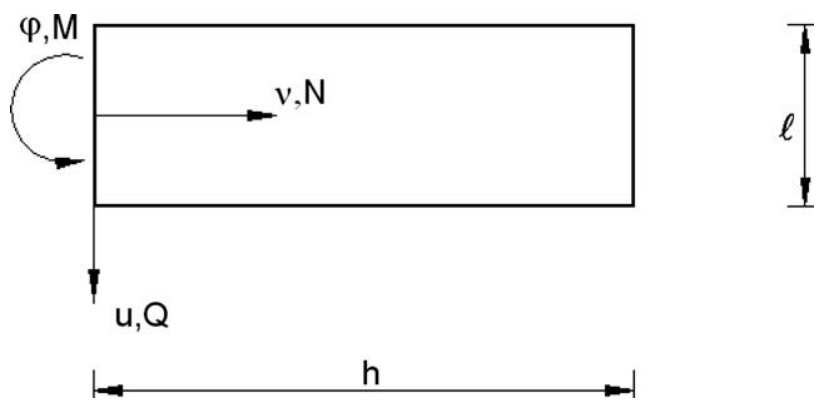

Fig. 2. Kinematics and statics of a beam.

For the Timoshenko beam:

$M_{11}=\frac{12 E^{(b)} I}{(1+b) h^{3}}, \quad M_{34}=\frac{6 E^{(b)} I}{(1+b) h^{2}}, \quad M_{22}=\frac{E^{(b)} A}{h}$,

$M_{33}=\frac{E^{(b)} I(4+b)}{h(1+b)}, \quad M_{36}=\frac{E^{(b)} I(2-b)}{h(1+b)}$

where, $E^{(b)}$ is the Young's Modulus; $t^{(b)}, h$ and $l$ are respectively the thickness, the span and the height of the Timoshenko beam; $A=t^{(b)} l$ is the cross-section area; $I=t^{(b)} l^{3} / 12$ is the moment of inertia; $b=a E^{(b)} l^{2} / G^{(b)} h^{2}$ is the dimensionless parameter in Timoshenko beam theory; $G^{(b)}=E^{(b)} / 2\left(1+v^{(b)}\right)$ is the modulus of rigidity, where $v^{(b)}$ is the Poisson's ratio.

For the beam studied in [11]:

$M_{11}=\frac{G^{(b)} A}{h}, \quad M_{34}=\frac{G^{(b)} A}{2}, \quad M_{22}=\frac{E^{(b) \prime} A}{h}$,

$M_{33}=\frac{G^{(b)} A h}{4}+\frac{E^{(b) \prime} I}{h}, \quad M_{36}=\frac{G^{(b)} A h}{4}-\frac{E^{(b) \prime} I}{h}$

where $E^{(b) \prime}=E^{(b)} /\left[1-\left(v^{(b)}\right)^{2}\right]$.

The relationship between the lattice and its continuum equivalent is obtained based on the equivalence of strain energy stored in a unit cell of a lattice with its continuum counterpart. The calibration results for a triangular GB lattice are listed as follows.

For the Euler-Bernoulli beam:

$E^{(b)}=\frac{E}{\sqrt{3}}\left(\frac{l}{h}\right)^{-1} \frac{t}{t^{(b)}} \frac{12+\left(\frac{l}{h}\right)^{2}}{4+\left(\frac{l}{h}\right)^{2}}, \quad v=\frac{4-\left(\frac{l}{h}\right)^{2}}{12+\left(\frac{l}{h}\right)^{2}}$

Note that the Poisson's ratio for Euler-Bernoulli beams is always zero.

For the Timoshenko beam:

$E^{(b)}=\frac{2}{\sqrt{3}}\left(\frac{l}{h}\right)^{-1} \frac{t}{t^{(b)}} \frac{E}{1-v}$,

$v^{(b)}=\frac{5(1+v)}{11(1-3 v)}-\frac{20}{11}\left(\frac{l}{h}\right)^{-2}-\frac{12}{11}$

For the beam studied in [11]:

$E^{(b)}=E, \quad v^{(b)}=v$

The stiffness matrix of a GB element is expressed as functions of material and geometry parameters of its three beams. Take the element shown in Fig. 3 as an example, let $R$ be the matrix relating the displacement vector $\mathbf{u}_{i j}=\left\{\begin{array}{llllll}u_{i} & v_{i} & \varphi_{i} & u_{j} & v_{j} & \varphi_{j}\end{array}\right\}^{\mathrm{T}}$ and $\mathbf{u}_{I J}=\left\{\begin{array}{llllll}u_{I} & v_{I} & \varphi_{I} & u_{J} & v_{J} & \varphi_{J}\end{array}\right\}^{\mathrm{T}}:$

$\mathbf{u}_{I J}=\mathbf{R} \mathbf{u}_{i j}$

Then the stiffness matrix of the GB element $\mathbf{K}$ can be expressed in the form 
a

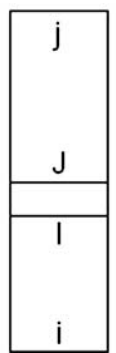

b

C

d

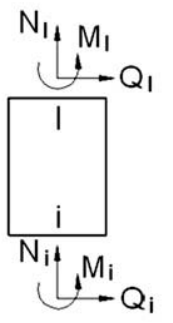

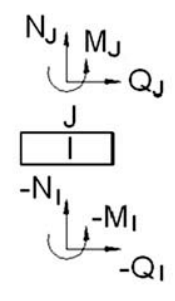

Fig. 3. A GB element composed of an aggregate beam, an interface beam and a matrix beam: (a) the GB element; (b) the aggregate beam; (c) the interface beam and $(d)$ the matrix beam.

$\mathbf{K}=\left[\begin{array}{cc}\mathbf{M}_{\mathbf{I}}^{\mathbf{a}}+\mathbf{M}_{\text {II }}^{\mathbf{a}} \mathbf{R}_{\mathbf{I}} & \mathbf{M}_{\text {II }}^{\mathbf{a}} \mathbf{R}_{\text {II }} \\ \mathbf{S Y M} & \left(\mathbf{M}_{\mathrm{II}}^{\mathbf{m}}\right)^{\mathrm{T}} \mathbf{R}_{\mathrm{III}}+\mathbf{M}_{\mathrm{III}}^{\mathbf{m}}\end{array}\right]$

where, the superscript of $\mathbf{M}$ declares the material property; In other words, $\mathbf{M}^{\mathbf{m}}, \mathbf{M}^{\mathbf{a}}$ and $\mathbf{M}^{\mathbf{i}}$ are respectively the stiffness matrix of matrix, aggregate and interface beams. $\mathbf{M}_{\mathbf{I}}^{\mathbf{a}}, \mathbf{M}_{\text {II }}^{\mathbf{a}}$ and $\mathbf{M}_{\text {III }}^{\mathbf{a}}$ denote the top-left, top-right and bottom-right $3 \times 3$ sub-matrix of $\mathbf{M}^{\mathbf{a}}$ respectively, and the same notation rule is also used to $\mathbf{M}^{\mathbf{i}}$ and $\mathbf{M}^{\mathbf{m}}$. $\mathbf{R}_{\mathbf{I}}$, $\mathbf{R}_{\text {II }}$ and $\mathbf{R}_{\text {III }}$, respectively denote the top-left, top-right, bottom-right $3 \times 3$ sub-matrix of $\mathbf{R}$.

It can be proved that the matrix $\mathbf{K}$ generally has the following distribution of non-zero elements:

$\mathbf{K}=\left[\begin{array}{cccccc}K_{11} & 0 & -K_{34} & -K_{11} & 0 & -K_{46} \\ & K_{22} & 0 & 0 & -K_{22} & 0 \\ & & K_{33} & K_{34} & 0 & K_{36} \\ & & & K_{11} & 0 & K_{46} \\ & & S Y M & & K_{22} & 0 \\ & & & & & K_{66}\end{array}\right]$

It can be found that $\mathbf{K}$ in (10) has more independent elements than $\mathbf{M}$ in (1) for the reason that a GB element has a more complex interstructure shown as Fig. 3. For the sake of convenience, the following matrix $\mathbf{K}_{N}$ is defined as

$\mathbf{K}_{\mathrm{N}}=\left[\begin{array}{cccccc}0 & 0 & 0 & 0 & 0 & 0 \\ & K_{22} & 0 & 0 & -K_{22} & 0 \\ & & 0 & 0 & 0 & 0 \\ & & & 0 & 0 & 0 \\ & S Y M & & & K_{22} & 0 \\ & & & & & 0\end{array}\right]$

If the computational effort is the main choke point in the simulation, it is suggested to use the GB lattice in which every aggregate is modeled by a single node [9]. Otherwise, if the size of the simulated specimen is not very large and the main aim is to observe the failure process in more details, another kind of GB lattice is preferred, in which a single aggregate covers dozens of nodes [10]. The simulations in this paper are mainly based on the latter. Furthermore, every GB element is composed of three beams of the same span and the same depth, which are all described by the Timoshenko beam theory.

Mohr-Coulomb theory is a mathematical model describing the response of materials such as rock, rubble piles or concrete to shear stress as well as normal stress. Most of the classical engineering materials somehow follow this rule in at least a portion of their shear failure envelope. The criterion can be expressed in the form

$|\tau|<c-\sigma \tan \phi$

where $c$ is the cohesive strength and $\phi$ is the friction angle; $\tau$ and $\sigma$ are the shear stress and the normal stress respectively.

Because materials such as concrete/rock have a very low tensile strength $f_{\mathrm{t}}$ as compared with their compressive strength and shear strength, the Mohr-Coulomb strength surface with tension cut-off [11] is adopted here (Fig. 4). Concrete's compressive strength $f_{\mathrm{c}}$ is generally 10-20 times the value of its tensile strength, so $f_{\mathrm{c}}=10 f_{\mathrm{t}}$ is used in this paper. Furthermore, the failure surface in Fig. 4 can be expressed by the following three inequations:

$$
\begin{aligned}
& \sigma<f_{\mathrm{t}} \\
& |\tau|<c-\sigma \tan \phi \\
& \sigma>-f_{\mathrm{c}}
\end{aligned}
$$

Then the implementation of the Mohr-Coulomb criterion shown in Fig. 4 is introduced. It is notable that we find out the critical element by checking the stresses in beams of GB elements. The normal stress can be expressed in the form [7]

$\sigma=\frac{N}{A}+\alpha \frac{\left(\left|M_{i}\right|,\left|M_{j}\right|\right)_{\max }}{W}$

where, $N$ is the normal force in the considered beam, $M_{i}$ and $M_{j}$ are the bending moments at the nodes $i$ and $j$ of the beam, and $W=t^{(b)} l^{2} / 6$ is the section modulus. The coefficient $\alpha$ regulates what part of the bending moment is considered. Lilliu and van Mier [12] have shown that simulation results are also satisfactory in the case of $\alpha=0$ though $\alpha$ is usually set to 0.005 [7-9]. Therefore, $\alpha$ is also set to zero in this paper. Another advantage of $\alpha$ being zero lies in that three beams in every GB element have the uniform normal stress because both $N$ and $A$ are uniform throughout every GB element.

From (1), the shear force can be expressed in the form

$Q_{1}=-Q_{2}=M_{11}\left(u_{1}-u_{2}\right)-M_{34}\left(\varphi_{1}+\varphi_{2}\right)$

In consideration of the equilibrium conditions at $I$ and $J$ (Fig. 3), it can be found that the shear force is also uniform throughout the GB element. Therefore, the shear stress can be calculated as

$|\tau|=\frac{\left|Q_{1}\right|}{A}=\frac{1}{A}\left|M_{11}\left(u_{1}-u_{2}\right)-M_{34}\left(\varphi_{1}+\varphi_{2}\right)\right|$

As a result, the efficiency of the numerical procedure is improved. Furthermore, it makes the following assumption reasonable: when some GB element becomes critical, it cracks into two fragments of the same span, i.e., $L / 2$, where $L$ is the length of the GB element. Therefore, if all six GB elements starting from node $i$ have failed, the isolated material domain around node $i$, called

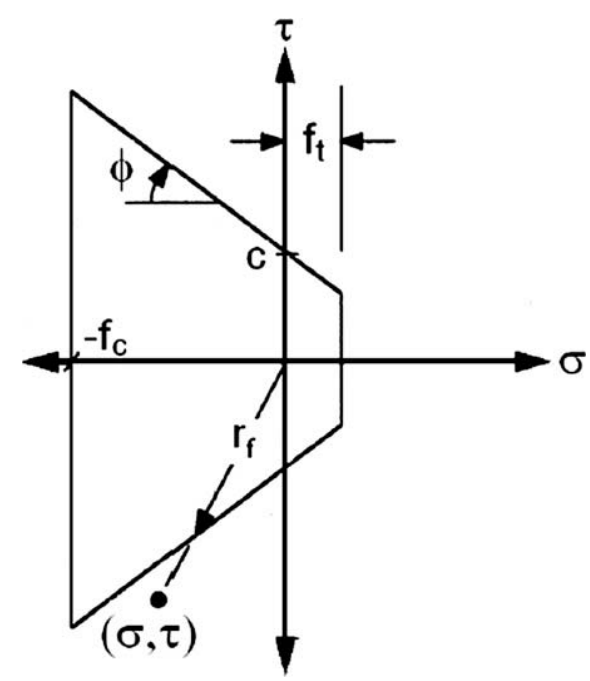

Fig. 4. Mohr-Coulomb strength surface with tension cut-off. 
influence zone of node $i$ has the geometry shown as Fig. 5a. The GB lattice can be also taken as a network by gluing a large amount of this kind of material domains, shown as Fig. 5b, where the short line-segment crossing the middle of every GB element indicates the potential crack path.

Then an important approximation is made: the material domain around every node is circular and its diameter is $L$, which is the same as the bonded-particle model (BPM) [14]. The advantages of the GB lattice over the BPM will be discussed later. When calibrating the GB lattice [9], it is assumed that the influence zone (unit cell) is hexagonal, which is $\pi / 2 \sqrt{3}$ times the area of the circle (Fig. $5 \mathrm{c}$ ). This difference can be avoided easily when using the equivalence of strain energy between the GB lattice and its continuum counterpart. But this difference is neglected in this paper. On one hand, it does not influence the results seriously. On the other hand, even though the model is a very rough approximation of reality, this appears throughout all kinds of lattice-type approaches [12].

The adoption of circular influence zones (unit cells) brings the following two advantages at least:

- The contact detection becomes very simple. To judge if contact happens in a cracked element, we only need to compare the initial length of the element with the current distance between its two nodes. Although only cases of small displacements are studied here, the circular-influence-zone assumption can also simplify the contact/separation detection when large displacements are permitted.

- The direct calculation of the stiffness matrix of recurred elements, i.e., contact elements, becomes possible. If the crack path in an element is arbitrary, the corresponding contact stiffness can not be calculated directly in principle without any assumption. There are mainly two kinds of assumptions: one takes the influence zones as rigid bodies [14], while the other assumes that the stress field throughout every special influence zone is uniform [15]. As shown in Fig. 5d, when the cracked element $i j$ contacts, the stiffness matrix of the contact element can be calculated directly because the properties of the two "half GB elements" are known. In this paper, we only study the simplest case-smooth contact. Therefore, the contact element can only bear compressive actions along the direction of element $i j$, and the stiffness matrix can be expressed in the form

$\mathbf{K}_{\text {contact }}=(1-D) \mathbf{K}_{\mathrm{N}}$

where, $D$ is the damage factor due to the deformation history. Of course, the concept of the damage factor here is extremely rough as compared with the one in the classical damage mechanics which increases gradually with external loads [16]. However, $D$ in (17) also indicates the degradation of material properties, so basically has the same physical meaning of the classical damage factor.

The above can be taken as a systemic sum-up of the GB lattice model.

Although lattice-type beam lattices have been mainly applied to investigate properties of particle composites until now, the basic construction of lattice modeling indicates that other kinds of inclusion can be also projected on to the lattice except particles. Meanwhile, concretes employed in practical engineering do contain many other inclusions, such as fiber and reinforcing rebar. Therefore, lattice modeling of reinforced fiber/particle concrete is an easy-doing but interesting task.

Fibers and rebar can be projected on to the matrix-phase lattice directly. The parts overlain by these inclusions are assigned the corresponding material properties. As shown in Fig. 6, the fibers are distributed in three directions with $60^{\circ}$ from each other. Of course, fibers can also be placed on other directions, but we argue that the fiber distribution in Fig. 6 leads basically to a macro isotropic medium with the similar reason that a regular triangular lattice corresponds to an isotropic continuum [8].

\subsection{Event-driven method}

There are mainly two kinds of numerical algorithms for simulating fracture process in heterogeneous brittle media such as concrete: event-driven method [10,11] and load-stepping method [13]. In the former, the load discretization is adjusted to capture every elemental failure. While in the latter, the load discretization is arbitrarily fixed, and we can deal with a great number of failure events during a single load step.

Then the differences between these two methods are being discussed. As commonly accepted, event-driven method is more accurate than load-stepping method. In the region passed through by crack, the stress level decreases substantially, preventing other elements in the crack's neighborhood from cracking. In other words, cracked element makes the other elements near to it less possible to fail any more. In the load-stepping method, more than one element fails in a single step, with an ignorance of the relative sequence of their failures, which will lead to very unrealistic results in some conditions. To show this problem more clearly, a one-dimensional fracture example is given as follows. A brittle bar is composed of six link elements as shown in Fig. 7a. These six elements have the same section-area, length and Young's modulus, but different tensile strengths $f_{\mathrm{t}}$, i.e., $2: 2$ : 1 : 1.001: 2 : 2. A quasi-static displacement-controlled tensile experiment is

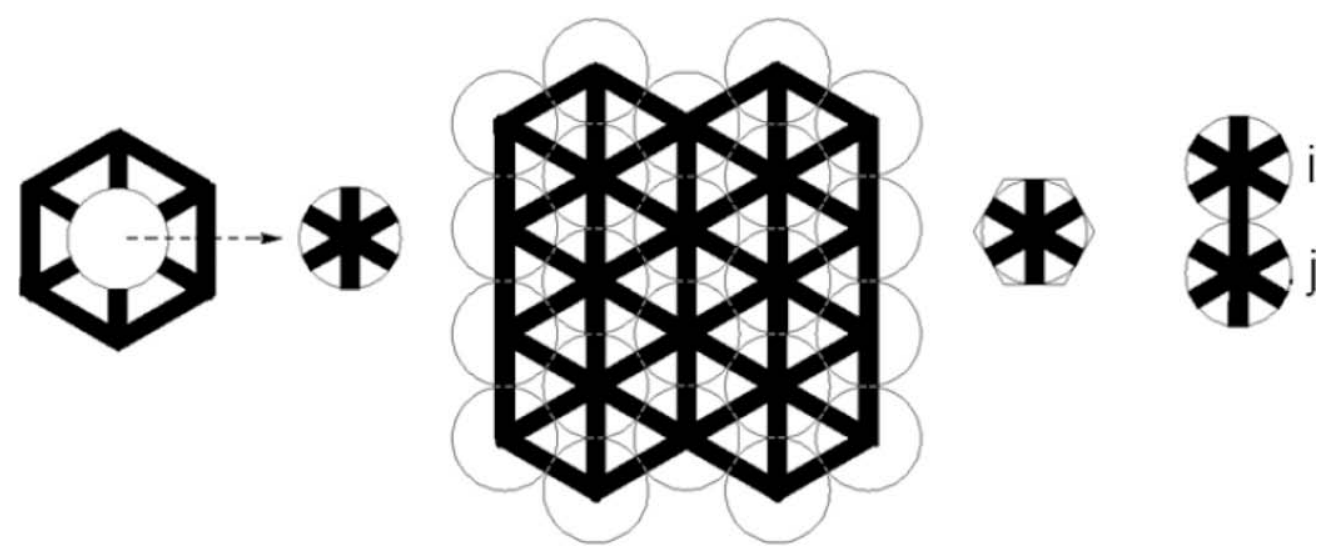

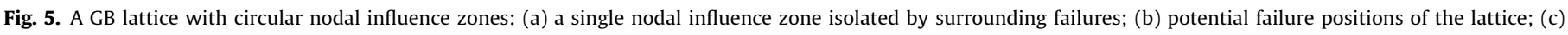
comparison of circular and hexagon influence zones and (d) sketch-map of contact. 


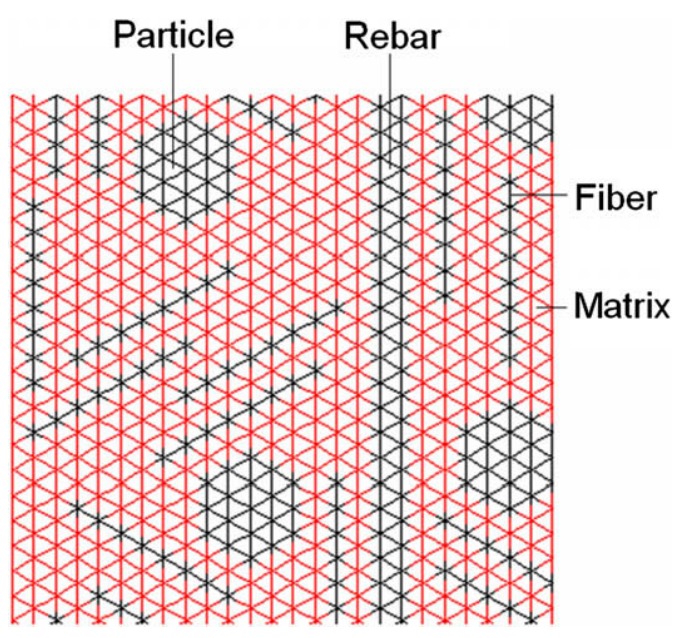

Fig. 6. GB lattice modeling for reinforced fiber/particle composites.
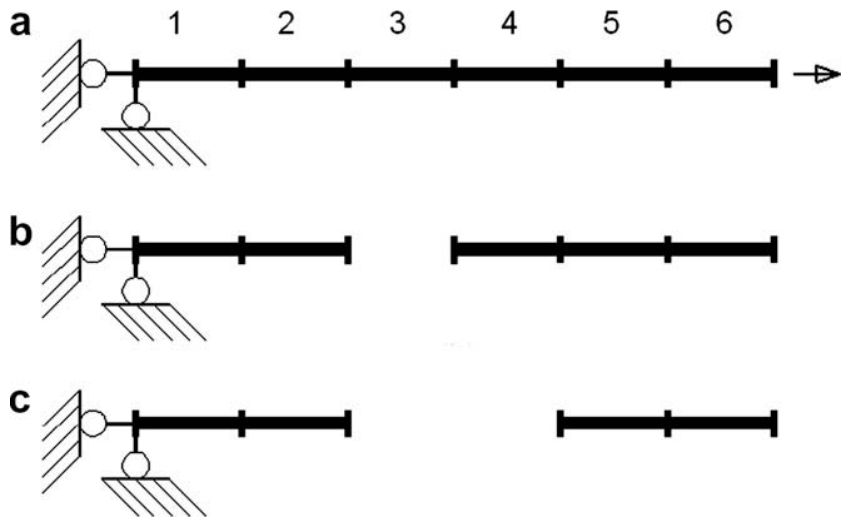

Fig. 7. Tensile experiment on a bar: (a) sketch map of the experiment set-up; (b) result for event-driven method and (c) result for load-stepping method when load step is not small enough.

conducted on the bar. It can be imagined that element No. 3 will fail and then all other elements return to zero-stress state. When using the event-driven method, element No. 3 has the biggest ratio of stress to strength, $R_{\mathrm{f}}=\sigma / f_{\mathrm{t}}$, so it undergoes the fracture event. The reasonable final state is shown as Fig. $7 \mathrm{~b}$. When using the load-stepping method, the ratio of $R_{\mathrm{f}}=\sigma / \mathrm{f}_{\mathrm{t}}$ in element No.3 to that in No.4 is 1.001: 1, indicating that critical stresses in these two elements are extremely close. Therefore, it is extremely possible for these two elements to fail in the same step. If so, the final crack pattern obtained by this method becomes Fig. 7c, which is much different from the expected pattern in Fig. 7b and therefore obviously unreasonable. In principle, this question can be completely overcome only by adopting a controlled-displacement step smaller than $\left(f_{\mathrm{t}}^{(4)}-f_{\mathrm{t}}^{(3)}\right) L / E A$, which is an extremely small value due to $f_{\mathrm{t}}^{(4)}-f_{\mathrm{t}}^{(3)}$ being very small, i.e., $f_{\mathrm{t}}^{(3)} / 1000$. The interesting thing here is that the event-driven method obtains more realistic result but meanwhile costs much less computational effort than the loadstepping method. Of course, the advantage in computational effort of the load-stepping method can become very important in the presence of a great number of simultaneous failures [13].

In the present paper, the event-driven method is adopted and the implementation in the GB lattice procedure is introduced as follows. Fracture is simulated by successive occurrences of "events", which may be failure of critical intact or partly failed elements, or contact/separation of former cracked elements. Then, the essential of numerical simulation is to detect new event(s) cor- rectly. The value of load increment in every step depends on the appearance of new critical element(s). After a trial load increment is applied, the normal stress $\sigma$ and the shear stress $\tau$ acting in each beam are compared with the fracture surface, the criterion for fracture is

$R=\frac{r}{r_{\mathrm{f}}}>1$

where $r=\left(\sigma^{2}+\tau^{2}\right)^{0.5}$ and $r_{\mathrm{f}}$ is as defined in Fig. 4. Analogously, the criterion for separation/contact of cracked elements is

$R=\frac{\sqrt{(\Delta u)^{2}+(L+\Delta v)^{2}}}{L}>1($ contact $\rightarrow$ separation $)$
$R=\frac{L}{\sqrt{(\Delta u)^{2}+(L+\Delta v)^{2}}}>1($ separation $\rightarrow$ contact $)$

where, $\Delta u=u_{j}-u_{i}$ and $\Delta v=v_{j}-v_{i}$ are relative displacements between nodes $i$ and $j$ in element $i j$ (Fig. 5d). As for the critical element, the update of stiffness matrix and the release of internal forces are introduced as follows:

(1) If (13a) is violated, $\left\{Q_{i} N_{i} M_{i} Q_{j} N_{j} M_{j}\right\}^{\mathrm{T}}$ is released, and the element stiffness matrix $\mathbf{K}$ is set to zero. It is notable that this element is definitely under tensile state in the current step. However, this element is possible to recur due to contact in the following steps. Once contact happens, the recurred stiffness matrix is

$\stackrel{\mathrm{T}}{\mathbf{K}}=(1-\stackrel{\mathrm{T}}{\mathrm{D}}) \mathbf{K}_{N}$

where, the superscript "T" in $\stackrel{\mathrm{T}}{\mathbf{K}}$ and $\stackrel{\mathrm{T}}{\mathrm{D}}$ represents "Tension". Thus, $D$ denotes the damage factor due to the failure by violating (13a), i.e., maximum tensile stress criterion. $\mathbf{K}_{N}$ has been defined in (11).

(2) If (13b) is violated, there are two possible cases. In one case, the normal stress $\sigma$ acting in the critical element is positive, i.e., tensile stress, so the element is not possible to become contact at the very beginning of stress redistribution. As a result, $\left\{Q_{i} N_{i} M_{i} Q_{j} N_{j} M_{j}\right\}^{\mathrm{T}}$ is released, and the element stiffness matrix $\mathbf{K}$ is set to zero. In the other case, $\sigma$ is negative, i.e., compressive stress, so the critical element becomes contacting once violation happens. Then, $\left\{Q_{i} \stackrel{\mathrm{MC}}{D} N_{i} M_{i} Q_{j}\right.$ $\left.{ }_{D}^{\mathrm{MC}} N_{j} M_{j}\right\}^{\mathrm{T}}$ is released, and the stiffness matrix is updated as $\stackrel{\mathrm{MC}}{\mathbf{K}}=(1-\stackrel{\mathrm{MC}}{D}) \mathbf{K}_{N}$

where the superscript "MC" stands for "Mohr-Coulomb". $\stackrel{\mathrm{MC}}{D}$ denotes the damage factor due to the failure under the actions of both the shear stress and the normal stress, defined as Mohr-Coulomb criterion in (13b).

(3) Three kinds of elements are possible to violate (13c): intact elements, elements damaged by violating (13a) previously, and elements damaged by violating ${ }_{c}(13 b)$ previously. As for intact elements, $\left\{Q_{i} D N_{i} M_{i} Q_{j} D N_{j} M_{j}\right\}^{\mathrm{T}}$ is released, and the stiffness matrix is updated as

$\stackrel{\mathrm{C}}{\mathbf{K}}=(1-\stackrel{\mathrm{C}}{\mathrm{D}}) \mathbf{K}_{\mathrm{N}}$

where the superscript " $\mathrm{C}$ " represents "Compression". As for the second kind of elements, $\left\{Q_{i}(D-D) N_{i} /(1-D) M_{i} Q_{j}\right.$ $\left.(\stackrel{\mathrm{C}}{D}-\stackrel{\mathrm{T}}{D}) N_{j} /(1-\stackrel{\mathrm{T}}{D}) M_{j}\right\}^{\mathrm{T}}$ is released, and the stiffness matrix is set to $\mathbf{K}$. As for the third kind of elements, $\left\{Q_{i}(\stackrel{C}{D}-\stackrel{M}{D}) N_{i} /(1-\stackrel{M}{D}) M_{i} Q_{j}(D-\stackrel{\text { MC }}{D}) N_{j} /(1-\stackrel{\text { MC }}{D}) M_{j}\right\}^{\mathrm{T}}$ is released, and the stiffness matrix is also set to $\stackrel{\mathrm{K}}{\mathbf{K}}$. 
Table 1

The micro elastic and strength properties of phases

\begin{tabular}{llcccll}
\hline & $E(\mathrm{Mpa})$ & $f_{\mathrm{t}}(\mathrm{Mpa})$ & $f_{\mathrm{c}}(\mathrm{Mpa})$ & $c(\mathrm{Mpa})$ & $\phi\left(^{\circ}\right)$ & $D$ \\
\hline Aggregate & 70,000 & 10.0 & 100.0 & 15.0 & 45 & 0.95 \\
Interface & 25,000 & 1.25 & 12.5 & 1.875 & 45 & 0.95 \\
Matrix & 25,000 & 5.0 & 50.0 & 7.5 & 45 & 0.95
\end{tabular}

In the table, $D=\stackrel{T M C}{D} \stackrel{\stackrel{C}{D}}{D}[10]$, and aggregate stands for particle/fiber phase.

It can be found that the above numerical algorithm is much more complex than that for simulations of tensile failures [7-9], due to the inclusion of separation/contact phenomena and the consideration of shear stress, which is reasonable for investigating more general failures instead of only tensile failures.

\section{Numerical examples and discussions}

Particle composites have been studied before by the GB lattice model. The main extension made here is the inclusion of fibers and rebar into numerical concrete, i.e., GB lattices. Therefore, more attentions are put to analyses of influences of fibers and rebar. As a result, numerical examples are provided in three levels: fiber composites in Section 3.1, fiber/particle composites in Section 3.2 and reinforced fiber/particle composites in Section 3.3. Six examples called case 1-6 are provided, successively.
In these examples, the employed GB lattice has a total of 8082 nodes and 23861 elements, and a rectangular geometry of 9 by $66 \sqrt{3} / 5 \mathrm{~cm}$. All elements are $\sqrt{3} / 10 \mathrm{~cm}$ long. The depth-to-span ratio is set to 1.0 for all beams. The micro elastic and strength properties for all examples except case 3 are shown as Table 1 .

\subsection{Tensile and compression experiments on fiber composites}

\subsubsection{Case 1: tensile experiment on fiber composites}

The sketch map for the tensile experiment is shown in Fig. 8a. The specimen contains 275 fibers. The load-displacement curve and crack patterns at typical load-levels can be seen in Fig. 8b-e, respectively.

In the tensile experiment, influences of the fibers' inclusion on the fracture process can be discussed as follows:

(1) The P- $\delta$ curve is divided into pre-peak regime and post-peak regime by the peak load (Fig. $8 \mathrm{~b}$ ). Directly following the peak load level, there is a steep drop in load, corresponding the formation of the macro failure band shown as Fig. 8c.

(2) Fibers parallel to load direction, i.e., $0^{\circ}$ fibers, do not improve the tensile loading capacity of the specimen. The reason is that elemental failures happen easily at ends of these fibers, preventing fibers from enduring larger tensile actions (Fig. $8 c)$
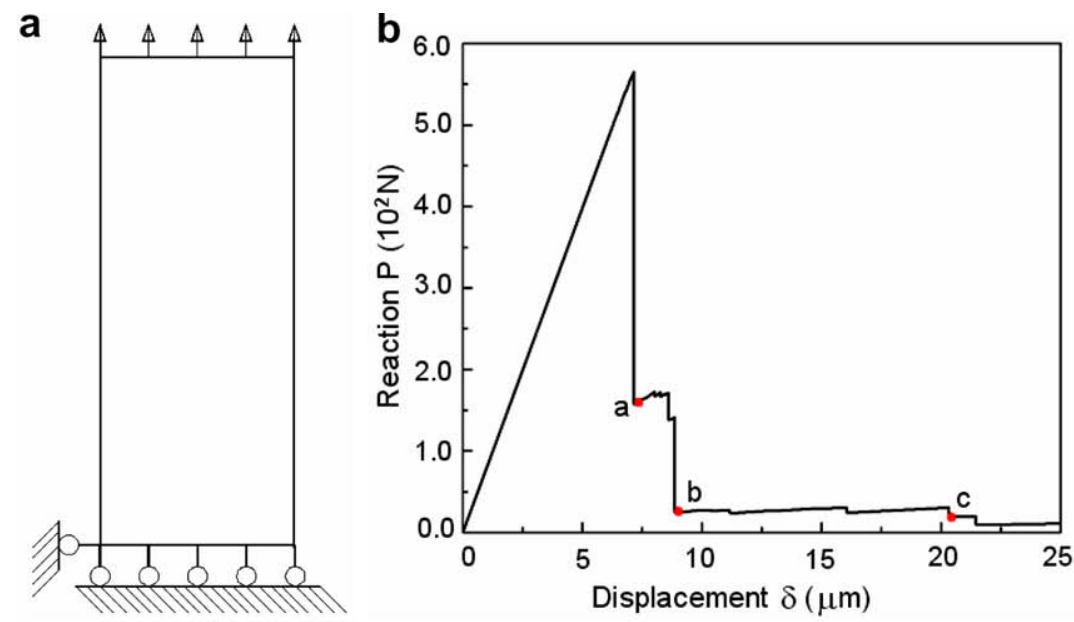

C

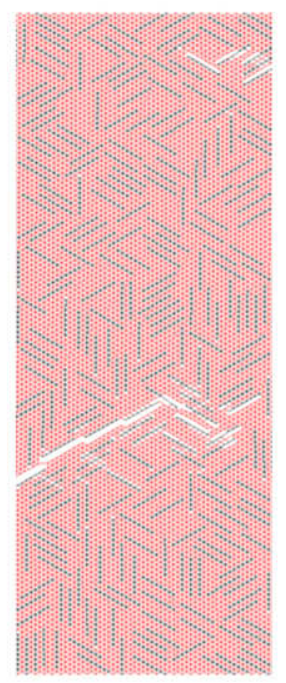

d

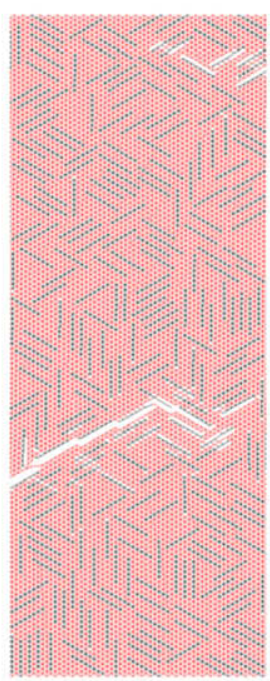

e

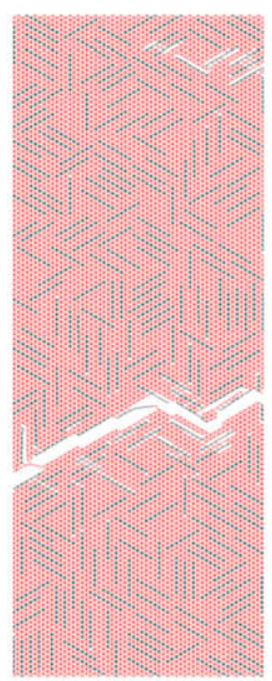

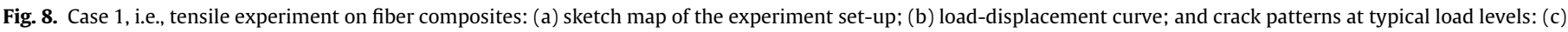
a; (d) b and (e) c. 
(3) Elements in the neighborhood of $\pm 60^{\circ}$ fibers are under tensile state, and are the dominant sources of failures because the interface phase between matrix and fibers is the weakest amid the three-phase system composed of matrix, inclusion (particle, fiber or rebar) and interface between them (Fib. $8 d$ ). The continuous interface zone surrounding the fiber somewhat acts as weak structural lines (face in 3D). When this kind of interface has failed, the degree of stress concentration on both ends of the interfaces is generally much highly than that in the case of particle composites because fibers have much bigger length-to-width ratio than particles (Fig. 6). Then it is expected that cracks extend more easily at fiber's ends. For this reason, matrix elements surrounding fibers that block micro-cracks' extensions may be more easily destroyed due to higher stress concentration.

(4) Crack-bridging happens frequently (Fig. 8c-e). "Bridges" between crack faces can be fibers and/or narrow matrix regions between fibers.

3.1.2. Case 2 and case 3: compressive experiments on fiber composites

The experiment set-up is shown as Fig. 9a. The specimen has the same fiber distribution as case 1 . Two cases are simulated: case 2 and case 3 . In case 2 , the material properties in Table 1 are employed. Their unique difference lies in that the fiber strength in case 3 is four times of that in case 2 .

Compressive failure is more complex than tensile failure because the former includes more micro-level failure mechanisms: compression-induced tensile failure, failure due to compression, and failure due to combination of normal and shear stresses [10].

In the present simulations, fibers have three kinds of directions: $0^{\circ}$ and $\pm 60^{\circ}$ relative to loading direction. In the pre-peak regime (Fig. 9b), many local cracks appear around $0^{\circ}$ fibers due to compression-induced tensile failure mechanism. These local cracks grow and join each other until the extension comes across $\pm 60^{\circ}$ fibers. $\pm 60^{\circ}$ fibers have two possible influences: blocking the cracks along $0^{\circ}$ fibers from extending in the original direction and transferring the extension to $\pm 60^{\circ}$; remaining the extension of cracks along $0^{\circ}$ fibers because of $\pm 60^{\circ}$ fiber breakage.

The fiber strength has an important influence on fracture processes. Case 3's specimen has higher fiber strength, so fiber breakage is more difficult to happen. As a result, crack extensions are blocked more easily by fibers: there is roughly a single macro failure band in case 2 (Fig. 9c), but several macro failure bands occur in case 3 during the post-peak regime (Fig. $9 \mathrm{~d}$ and e). Furthermore, the peak load increases with the fiber strength increasing (Fig. 9b).

\subsection{Case 4: compression experiment on fiber/particle composites}

The boundary conditions are the same as Fig. 9a. 170 fibers and 70 particles are distributed in the specimen. The results about the fracture process are shown in Fig. 10.

Firstly, the strain distribution is analyzed. In the pre-peak regime, many failure events whose sizes are much smaller than the specimen size happen mainly around fibers and particles
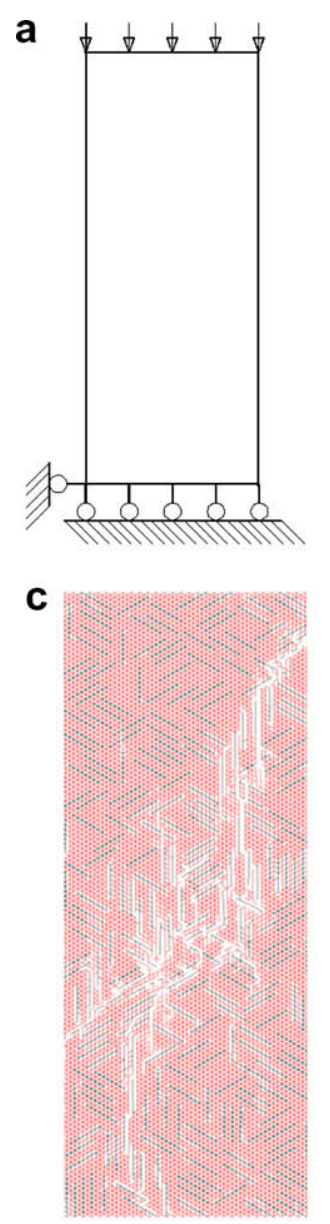

b
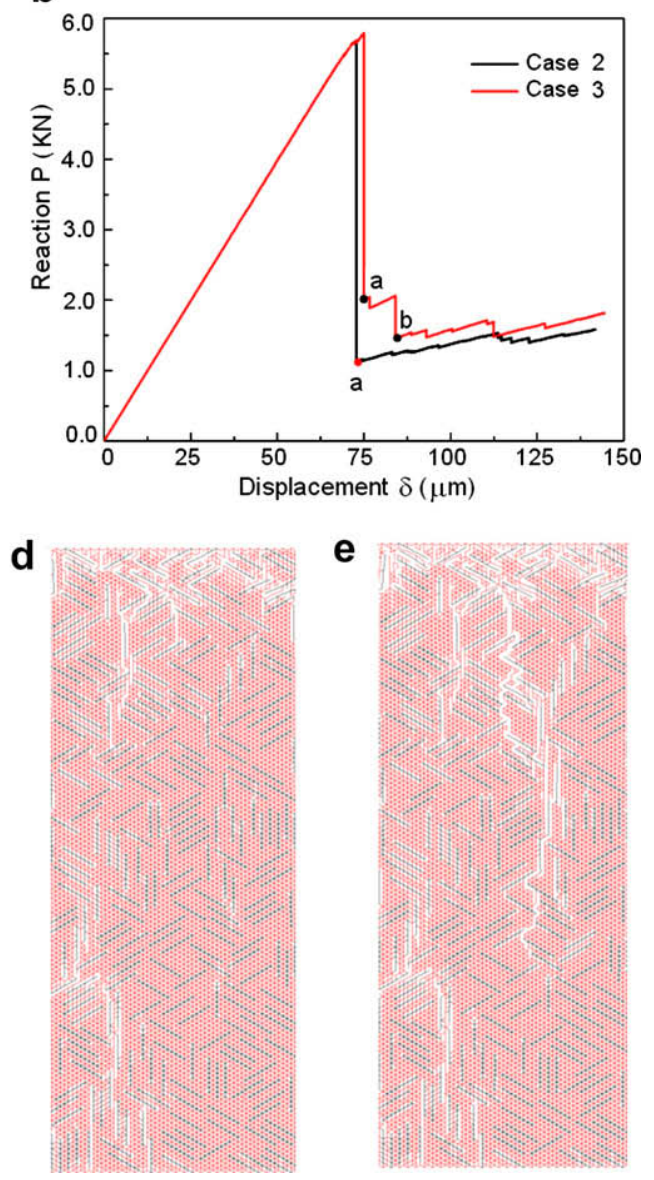

e

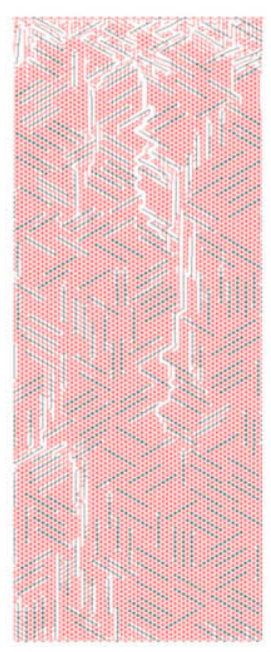

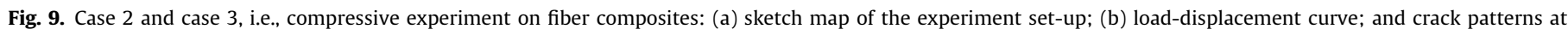
typical load levels: (c) a in case 2 ; (d) a in case 3 and (e) b in case 3. 
(Fig. 10b and c). From Fig. 10d, the strain field in the microstructure level is extremely unhomogeneous at the peak load level a. Meanwhile, however, it can be found that there is no macro strain localization. After the steep drop of load directly following the peak, an obvious strain localization band appears and deformations in other regions outside the band decrease to a much lower level (Fig. 10e and $\mathrm{f}$ ).

Before appearance of the localization band, the macro strain field can be regarded as uniform, even though the deformation in micro level is chaotic due to the micro-structural disorder. Failures at different positions nucleate and grow approximately independently. When the content of fiber/particle is fixed, the macro response is not very sensitive to the fiber/particle distribution. This indicates that the material is translationally invariant, therefore continuum damage mechanics can be a proper alternative to describe the pre-peak response [4]. More numerical investigations have shown the slopes of load-displacement curves are approximately common for specimens with different fiber/particle distributions, even though the corresponding numerical results have not been provided here for the sake of pithiness. However, crack patterns in the post-peak regime are strongly related to the inclusions' distribution, which dominates the position and propagation of the macro crack band.

Fracture history in the specimen can be observed from the view point of acoustic emission (AE), which is an important technique in real physics experiments. Fig. 10b shows the numerical AE curve. It can be found a majority of cracked elements fail during the steep drop directly following the peak load, and most of them localize in the macro crack band (Fig. 10e).

\subsection{Case 5 and case 6: three-point bending experiments on reinforced fiber/particle composites}

The experiment set-up is shown in Fig. 11a. In case 5, the specimen contained two pieces of rebar. The rebar's elastic property is the same as that of fiber/particle, while its strength is set so large that the rebar only deforms elastically and does not fail during the whole process. This operation seems reasonable because the rebar failure in realistic reinforce concrete is also hardly found. In case 6 , the specimen is the fiber/particle composites without rebar. The aim here is to highlight the influence of rebar inclusion on fracture process by comparing case 5 with case 6 :

(1) There is a much more complex pre-peak regime in case 5 , as shown in Fig. 11b. In the pre-peak regime, the load increases approximately monotonously with displacement in case 6 , but the P- $\delta$ curve in case 5 is anything but smooth, containing dozens of considerable steep drops.

(2) At the peak-load level, there are several macro cracks amid regions without rebar in case 5 (Fig. 11c), but no macro crack occurs in case 6 (Fig. 11f). In other words, the crack pattern in case 5 is more diffused that case 6 . Furthermore, case 5 has a
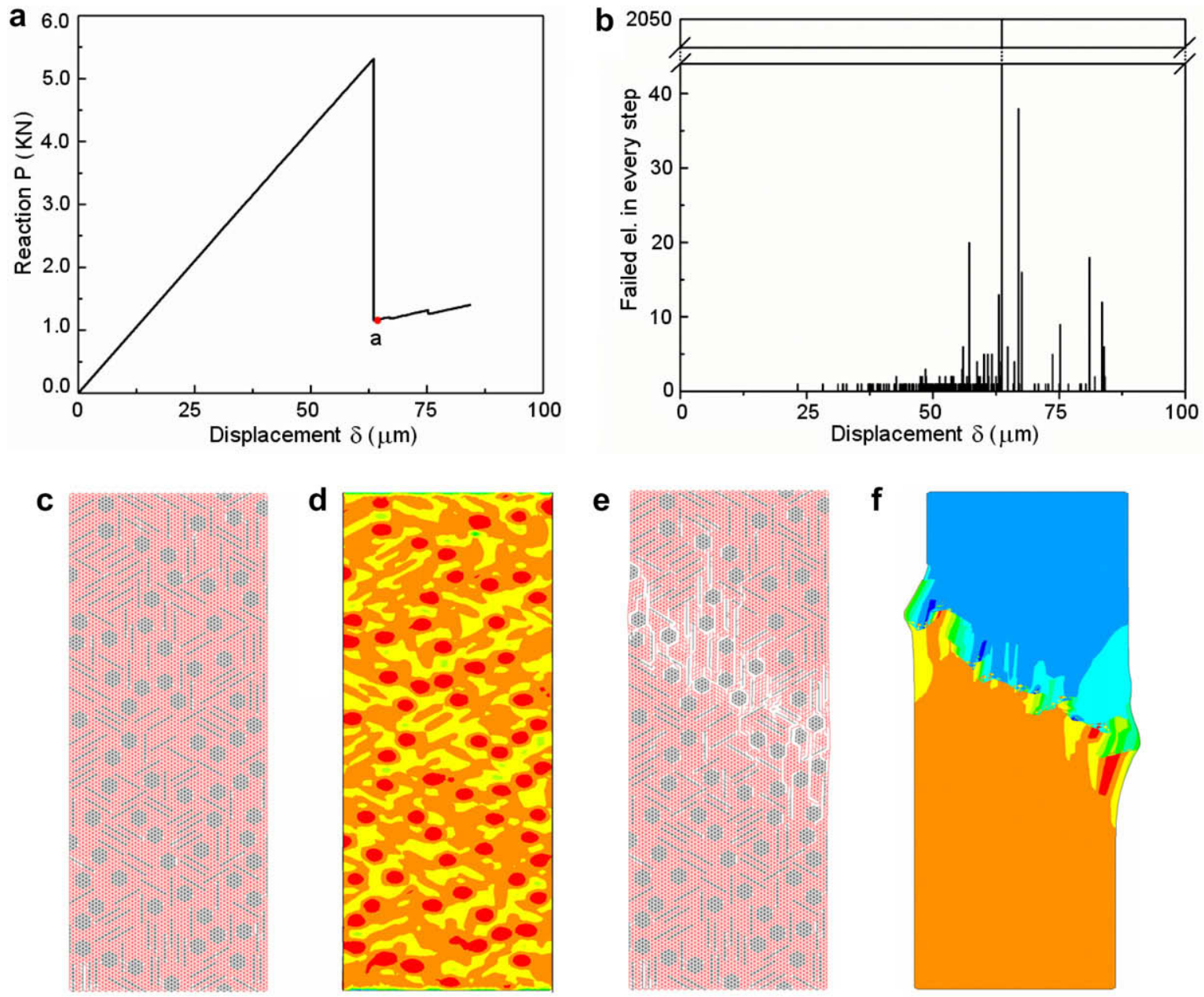

e

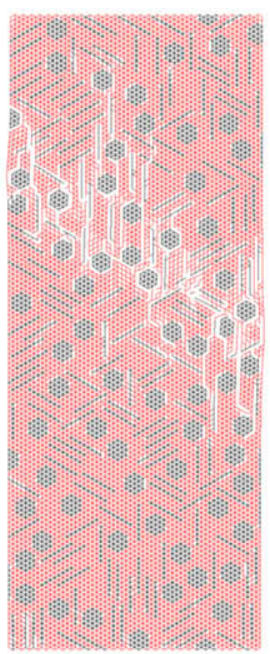

f

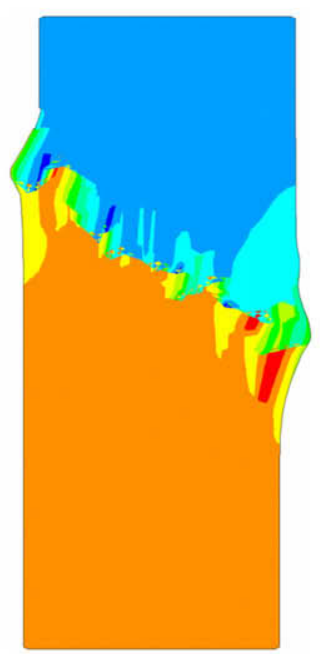

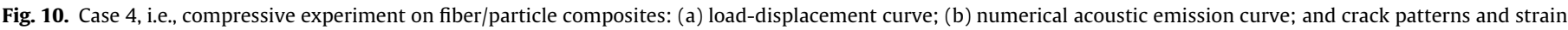
contours at typical load levels: (c) and (d) for a and (e) and (f) for b. 

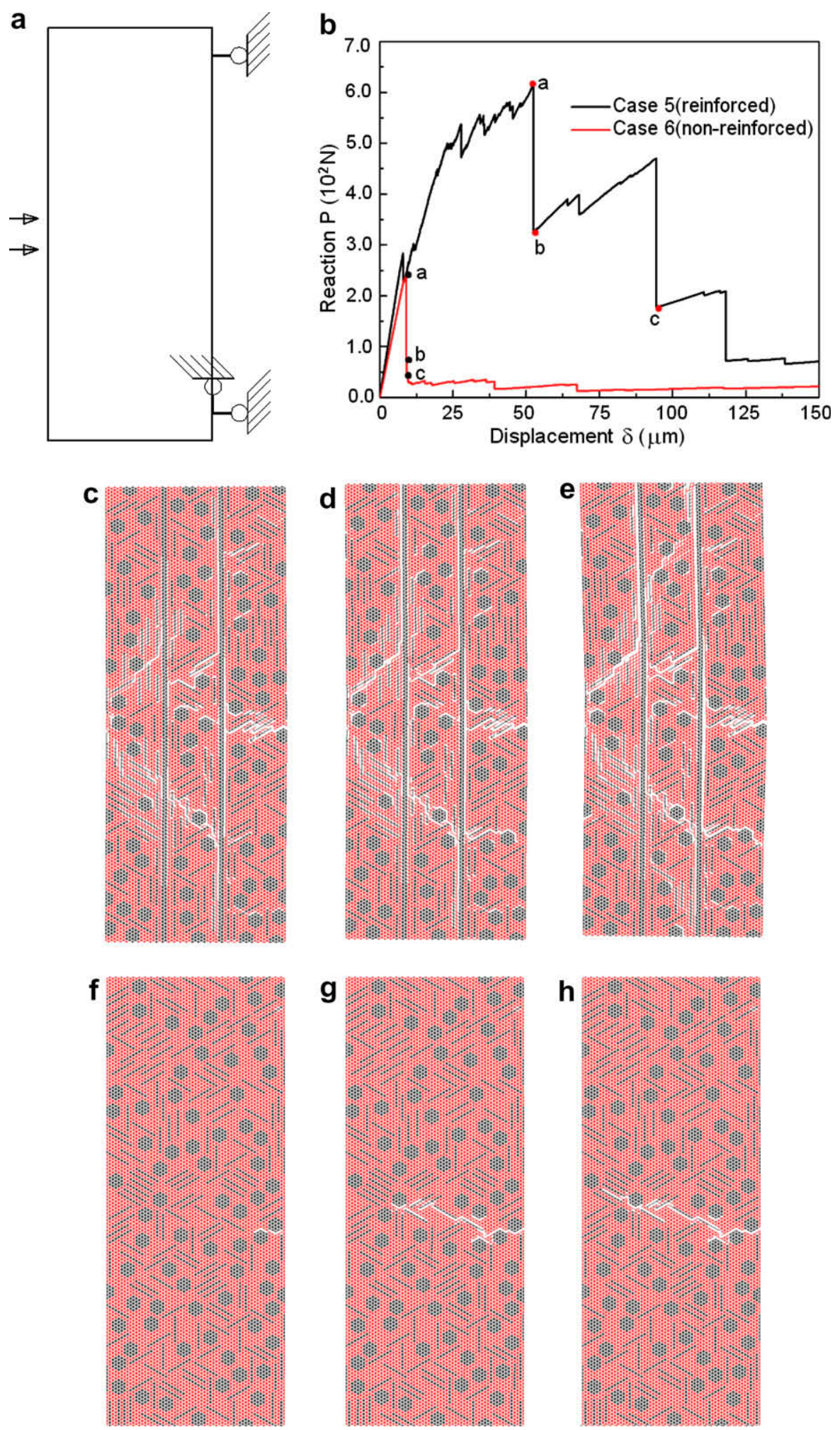

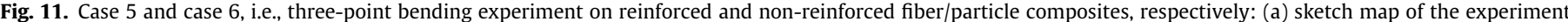

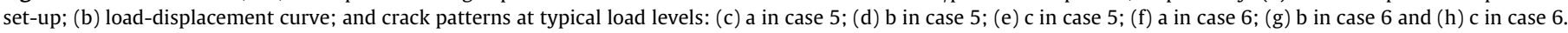

much higher peak load, indicating that rebar's inclusion can greatly improve the bearing capacity of load.

(3) The crack patterns are separated by the rebar into several parts in case 5 (Fig. 11c-e). When cracks come across rebar, its extension is prohibited. While in case 6 , the crack nearly grows along a single path (Fig. $11 \mathrm{f}-\mathrm{h}$ ). As a result, the ductility can also be improved by adding rebar into the composites (Fig. 11b).

\section{Conclusions}

The GB lattice model was extended into fracture analyses of reinforced fiber/particle composites. The inclusions in this kind of materials, i.e., fiber, particle and rebar are projected on to matrix-phase lattice directly. Therefore, the micro-level failure process can be observed more explicitly when compared with macro 
continuum models. In other words, the present model is suitable for analyses of micro-level failure mechanics.

In order to investigate the influence of fiber, particle and rebar on fracture behaviors, experiments were conducted on three kinds of composites respectively: fiber composites, fiber/particle composites and reinforced fiber/particle composites. Two new kinds of representation of fracture processes are given: strain contour and numerical acoustic emission curves, to highlight the physics phenomena as well as load-displacement curves and crack patterns.

\section{Acknowledgements}

This research work was sponsored by National Natural Science Foundation of China through Grant Nos. 10572140 and 10232050, and also partly by Ministry of Science and Technology Foundation No. 2002CB412706. The first author, Jinxing Liu, is greatly grateful to Dr. Xuejun Wang for his valuable discussions on the MohrCoulomb criterion, and to Dr. Xiaojiang Shang for his valuable discussions on general knowledge about concrete's nonlinear behavior.

\section{References}

[1] D. NgO, A.C. Scordelis, Finite element analysis of reinforced concrete beams, J. Am. Concrete Inst. 64 (1967) 152-163.

[2] Y.R. Rashid, Analysis of reinforced concrete pressure vessels, Nucl. Eng. Des. 7 (1968) 334-344.
[3] R. de Borst, J.J.C. Remmers, A. Needleman, M.A. Abellan, Discrete vs smeared crack models for concrete fracture: bridging the gap, Int. J. Numer. Anal. Meth. Geomech. 28 (2004) 583-607.

[4] D. Krajcinovic, M. Vujosevic, Intrinsic failure modes of brittle materials, Int. J. Solids Struct. 35 (1998) 2487-2503.

[5] T. Belytschko, T. Black, Elastic crack growth in finite elements with minimal remeshing, Int. J. Numer. Methods Eng. 45 (1999) 601-620.

[6] N. Moës, J. Dolbow, T. Belytschko, A finite element method for crack growth without remeshing, Int. J. Numer. Methods Eng. 46 (1999) 131-150.

[7] J.G.M. van Mier, M.R.A. van Vliet, T.K. Wang, Fracture mechanisms in particle composites: statistical aspects in lattice type analysis, Mech. Mater. 34 (2002) 705-724.

[8] B.L. Karihaloo, P.F. Shao, Q.Z. Xiao, Lattice modelling of the failure of particle composites, Eng. Fract. Mech. 70 (2003) 2385-2406.

[9] J.X. Liu, S.C. Deng, J. Zhang, N.G. Liang, Lattice type of fracture model for concrete, Theor. Appl. Fract. Mech. 48 (2007) 269-284.

[10] J.X. Liu, Z.Y. Zhao, S.C. Deng, N.G. Liang, Numerical investigation of crack growth in concrete subjected to compression by the generalized beam lattice model, Comput. Mech., doi:10.1007/S00466-008-0305-Z.

[11] J.E. Bolander, S. Saito, Fracture analysis using spring networks with random geometry, Eng. Fract. Mech. 61 (1998) 569-591.

[12] G. Lilliu, J.G.M. van Mier, 3D lattice type fracture model for concrete, Eng. Fract. Mech. 70 (2003) 927-941.

[13] A. Ibrahimbegovic, A. Depaplace, Microscale and mesoscale discrete models for dynamic fracture of structures built of brittle material, Comput. Struct. 81 (2003) 1255-1265.

[14] D.O. Potyondy, P.A. Cundall, A bonded-particle model for rock, Int. J. Rock. Mech. Min. Sci. 41 (2004) 1329-1364.

[15] G. Shi, Discontinuous deformation analysis - a new numerical model for the statics, dynamics of block systems. Ph.D. Thesis, University of California, Berkeley, USA, 1988.

[16] J. Lemaitre, A Course on Damage Mechanics, Science Press, Beijing, 1996 (in Chinese). 